\title{
CLINICAL CASE
}

\section{HYDATID CYST - A CHALLENGING DIAGNOSIS AND TREATMENT MATTER FOR THE YOUNG PRACTITIONER - THE MANAGEMENT OF A COMMON PATHOLOGY WITH FREQUENT COMPLICATIONS}

\author{
Oana Andreea Dobrin ${ }^{1}$, Andreea Frasin ${ }^{1}$, Elena Stanciu ${ }^{1}$, R. I. Toader ${ }^{1}$, D.N. Păduraru ${ }^{1,2}$, \\ Adriana Elena Nica ${ }^{1,3}$, C. V. Toma ${ }^{1,4}$, V.C. Coșei ${ }^{5}$ \\ ${ }^{1}$ The University of Medicine and Pharmacy "Carol Davila" Bucharest, Romania \\ ${ }^{2}$ III'rd Department of General Surgery, University Emergency Hospital Bucharest, Romania \\ ${ }^{3}$ Department of Anaesthesiology, University Emergency Hospital Bucharest, Romania \\ ${ }^{4}$ The Department of Urology, Clinical Hospital "Prof. Dr. Theordor Burghele" Bucharest, Romania \\ ${ }^{5}$ The Institute of Pneumology "Marius Nasta" Bucharest, Romania \\ Corresponding author: Dan Nicolae Păduraru \\ Phone no. 0040744756443 \\ E-mail: dan.paduraru.nicolae@gmail.com
}

\begin{abstract}
The hydatid cyst, or echinococcosis, is a parasitic disease whose etiologic agent is Echinococcus granulosus, a parasite pertaining to the Cestodes class. Most commonly, in humans, the disease is transmitted digestively, through the eggs of tapeworms found on the animal fur. In echinococcosis, the existence of an occupational risk for animal breeders was identified, which explains farming Romania as a hyperendemic country. The complicated clinical picture of Echinococosis may possess hurdles for the young practitioner, in his endeavor to establish a correct diagnosis. This paper presents the particular case of a 49-year-old man, diagnosed with lung hydatid cyst, without evidence of liver injury, even though the normal life cycle of the parasite involves liver passage. At first glance, the patient arrived in a hospital department complaining of febrile symptoms with purulent cough and chest pain that does not resonate with the clinical picture of a hydatid cyst, as was later discovered from anamnesis. From the patient's medical record, it stems that the patient refused surgical interventions, and followed an antiparasitic treatment for a year.In the eyes of a young practitioner, all this information hampers the differential diagnosis, delaying the decision of addressing the patient's problems through a surgery.In a general sense, depending on the organ that is affected, the most important part of the treatment of the hydatid cyst is represented by the surgical approach, which must be adapted to each case, taking into account the existing complications, the risks that may arise, the anatomic and pathologic substrate of the patient.The laparoscopic approach is preferable because of the aesthetic result, but in this case, a classical method was used, because of the high risk of complications during and after surgery.
\end{abstract}

Keywords: hydatic cyst, echinococcosis, Cestodes class, Echinococcus granulosus

\section{Introduction}

The hydatid cyst, or echinococcosis, is a parasitic disease whose etiologic agent is Echinococcus granulosus, a parasite pertaining to the Cestodes class. The hydatid disease is transmitted digestively, via E. granulosus eggs, directly from a dog's fur or the fur of animals in contact with the dog. The clinical manifestations depend on the size of the hydatid cyst and on 
the affected organ or tissue by the parasites. The most commonly affected organ is the liver (50$60 \%$ of the cases) and the second most common is the lung (30-40\%) [2]. According to a recent Romanian study, the liver is affected in a proportion of $84,7 \%$, out of which one-fifth of the patients experienced complications [1].

In terms of prevalence, Romania is amongst the countries with the highest values, higher than 5 cases per 100,000 inhabitants, according to a study carried between 1985 and 1997, by Iacobiciu et al [1] (Figure 1 and 2).

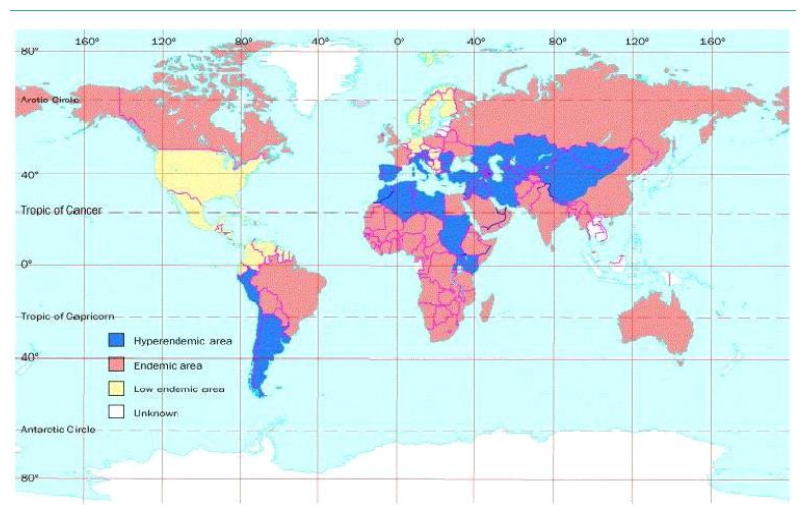

Figure 1 - Worldwide distribution of E. Granulosus

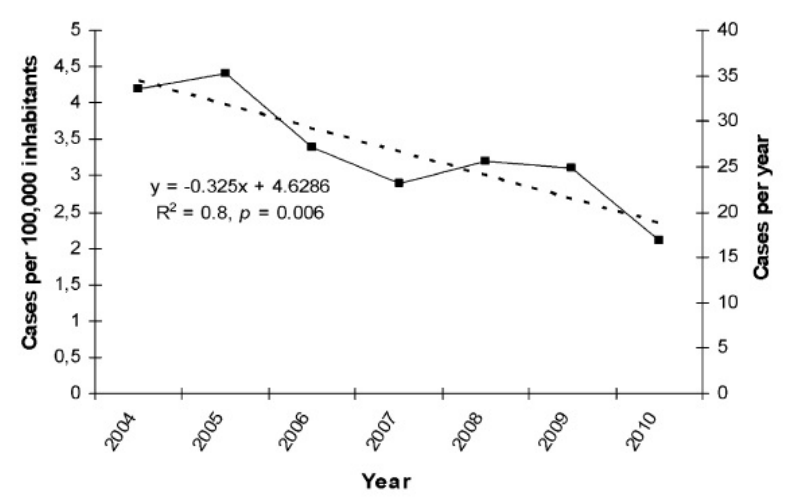

Figure 2 - Number of cystic echinococcosis cases and cases $/ 100,000$ persons in Caras-Severin and Hunedoara counties, Romania, 2004-2010 [1]

The clinical manifestations of the lung cyst are: dry cough, chronic irritation, and later on, small and repeated hemoptysis, chest pressure sensation, shortness of breath and vomica in case of bronchial opening of the cyst, with hydatid fluid disposal. Non-specific and minimal initial symptoms lead to a late reporting to the doctor of the patient, thus an early diagnosis is rarely made.

Associated to late symptoms, parapneumonic processes or pulmonary suppurations can occur as a result of the superinfection of the cyst, or atelectasis, which again raises difficulties in making a differential diagnosis.

The incubation time of the disease is unknown, but is considered long, 10-30 years, except when it is localized at the brain, eye, heart and musculoskeletal level, when the disease emerges fast, in 8-12 months [3]. The incidence of bone hydatid cyst varies between $0.5-4 \%$, according to various studies. Primary bone infection is very rare. The spine is the most frequent localization of infection, because the infection is done by extension relationship from the lung. In case bones are affected, the major symptom is pain, and the risk of fracture is higher.

Patients with cerebral hydatidosis usually report to the doctor with focal neurological deficit and symptoms of increased intracranial pressure. Cysts localized at the heart level occur at a rate of less than $2 \%$ of cases of echinococcosis and symptoms are: complete heart block, constrictive pericarditis and congestive heart failure [4].

Although the incidence of extrahepatic and extrapulmonary hydatidosis is low, its manifestations are major and life threatening. The conclusion is that the parasitosis may appear clinically polymorphously, which will improve the skills of a young practitioner, when it comes to observation and symptomatology analysis.

\section{Case presentation}

We present the case of a 49-year-old man, non-smoker, with relevant occupational exposure (animal farm) that reported to the doctor with the following symptoms: cough, eventually mucopurulent, fever (37.6 C), chills and right chest pain with acute onset, accompanied by loss of appetite and weight ( 2 $\mathrm{kg}$ ). From his medical history data, we know that the patient was diagnosed in 2013 with lung hydatid cyst, for which he refused surgery and received treatment with Albendazole between 2013-2014 and in 2015.

Objective clinical examination at admission into the hospital revealed no signs of other pathologies, the patient having a good general condition, bilateral pulmonary vesicular murmur with crackles detected in the middle third on the 
right side. Physical examination of the abdomen was normal, with the liver at the costal margin. Laboratory findings show leukocytosis with neutrophilis $(72.1 \%)$, normal percentage of eosinophils, hyperglycemia $(130 \mathrm{mg} / \mathrm{dl})$, slight increase in transaminases (TGP $=41 \mathrm{UI} / \mathrm{l}$, TGO $=62 \mathrm{UI} / \mathrm{l})$, with progressive evolution until Albendazole was interrupted, during hospitalization, at which moment the values normalized.

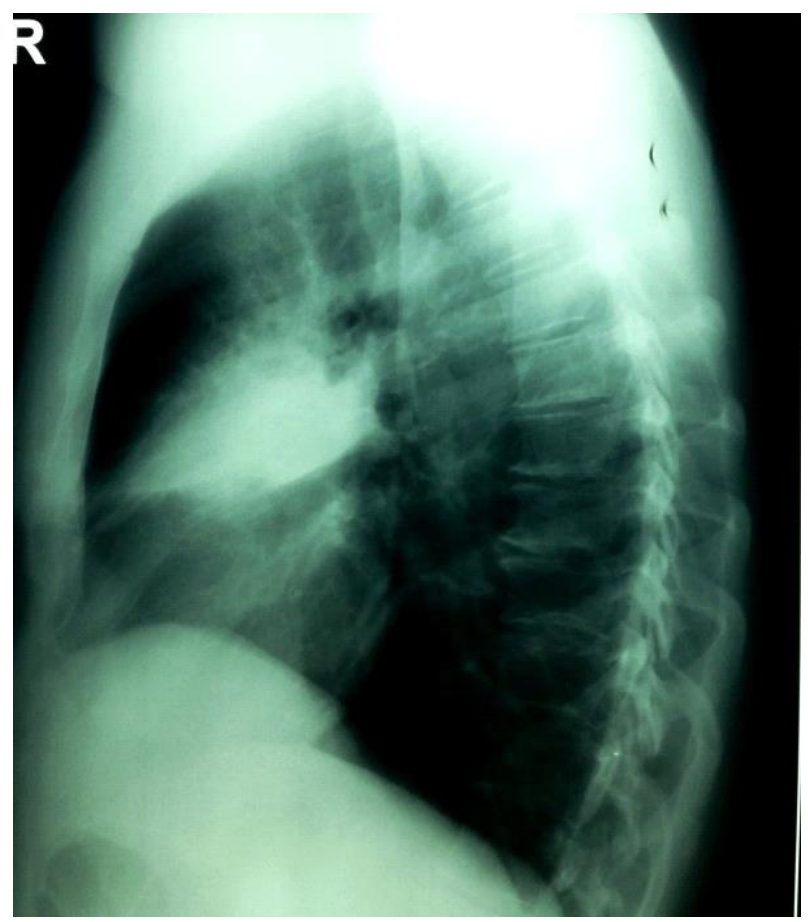

Figure 3 - Preoperative profile radiography

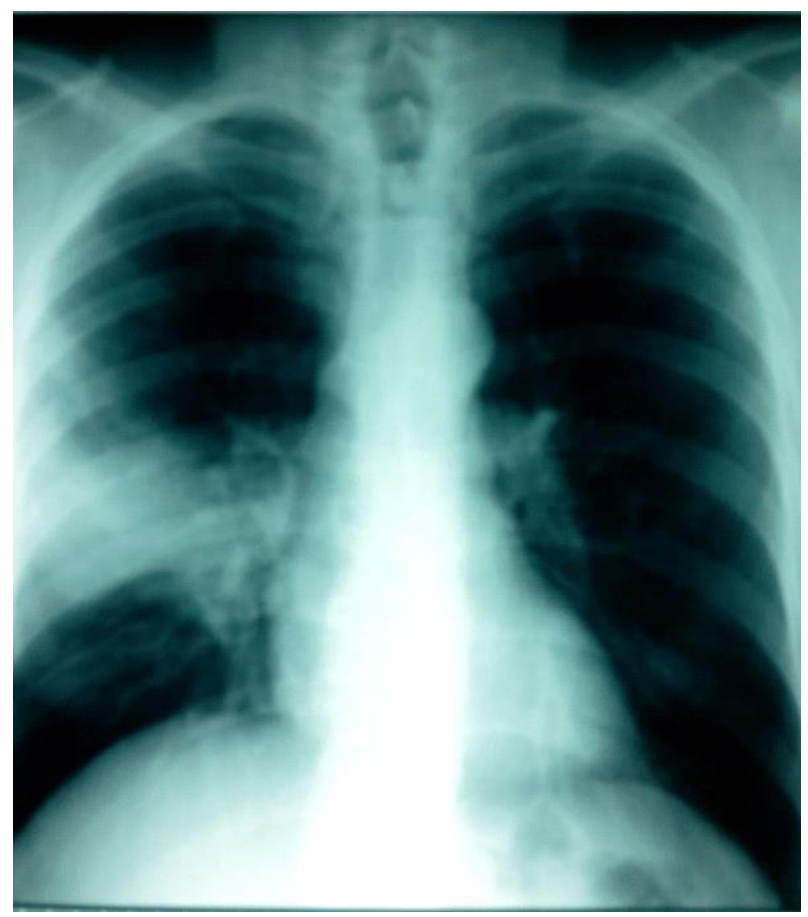

Figure 4 - First front Rx showing an opacity in the right lung field

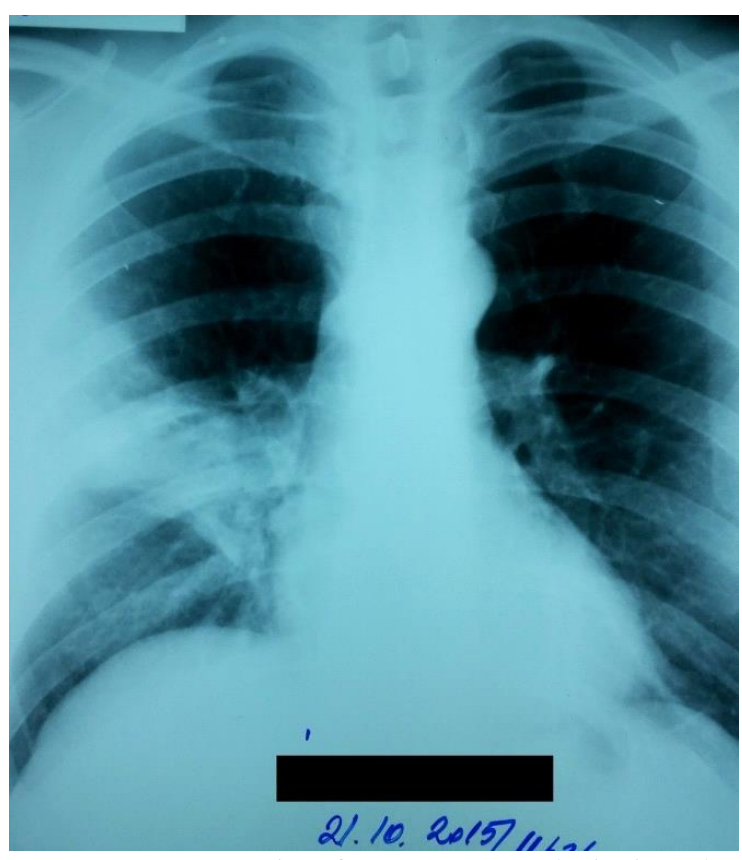

Figure 5 - Preoperative front $\mathrm{Rx}$, on admission day

Chest radiography image shows a thick-walled hollow, relatively triangular opacity, relatively well defined, localized in the lateral segment of the medium lobe, right lung field, supracostal intensity, heterogeneous through the presence of hypertransparent areas, with a relatively clear contour (Figures 3-5).

Thanks to an inconclusive appearance of the $\mathrm{x}$-ray, a CT is performed, confirming a process of pulmonary condensation on the right side, developing an abscess cavity, adjacent to the parietal pleura and mediastinal lymph nodes (Figure 6-7). These aspects lead the differential diagnosis to the existence of a pulmonary tumor, superinfection of a TB cavern or even the persistence of the hydatid cyst with extra pneumonic process, despite the treatment with Albendazole.

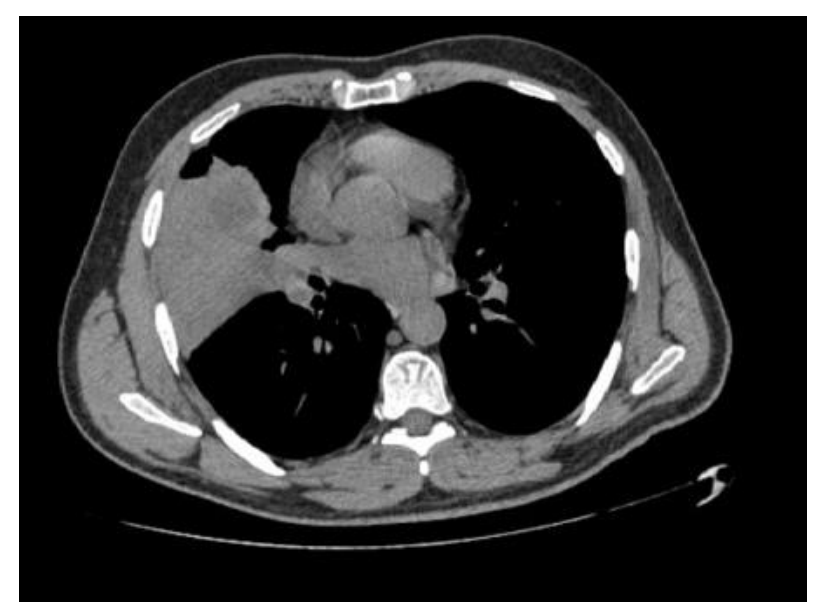

Figure 6 - CT scan without showing a condensation image 


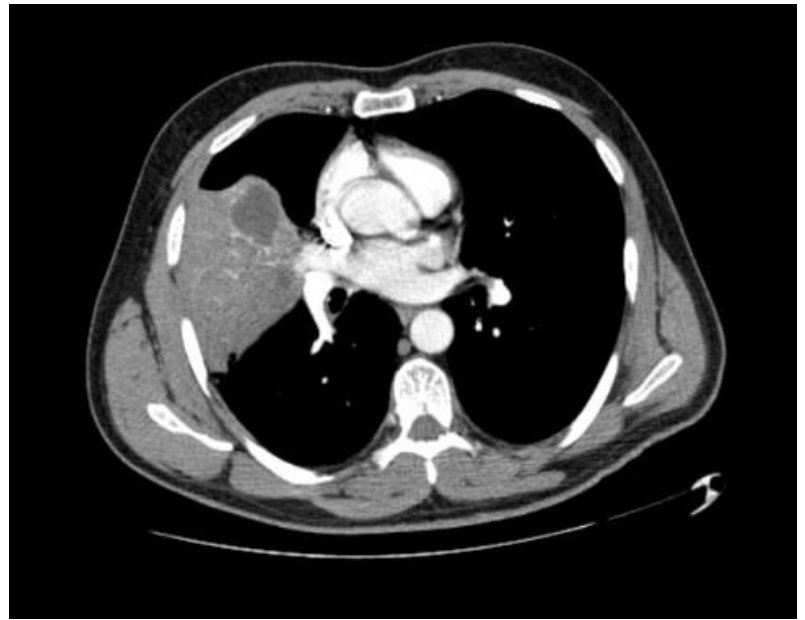

Figure 7 - CT scan with contrast substance showing a cavity

The bronchoscopy describes a purulent yellow-green sputum, with non-specific flora, without neoplastic cells, negative for Koch bacillus, excluding the aetiology of TB.

An empiric antibiotic treatment was initiated (as the bacteriological examination of the sputum was inconclusive for aerobic pathogenic bacteria) that lead to a stationary evolution, with persistent fever and chest pain.

Specific treatment was associated with anaerobic anti-bacteria.

During hospitalization, the patient had vomica in medium quantity, with clear liquid and salty-bitter taste, followed by a radiological and biological improvement of symptoms and of the clinical picture.

\section{Surgical approach}

Given the inconclusive aspect of the CT results and of the other laboratory explorations conducted in 2015, a thoracic surgery examination was requested, after which it was decided that the surgical approach excision has to be performed.

The procedure was performed under general anaesthesia, followed by an intubation with a double lumen endotracheal tube (Figure 8), in order to achieve the separation of the ventilation of lungs, with selective vent on the left side [5].

An incision in the right 5 IC area was performed. At that moment, there were two possibilities of open surgical approach: lobectomy or atypical resection.

Due to the localization of the cyst in the medium lateral lobe and due to the age of the patient, as well as to the indication of preserving as much lung tissue as possible [6], an excision via an atypical resection was decided. The atypical resection was made based on the edges of the lesion, disregarding the anatomy of the lung.

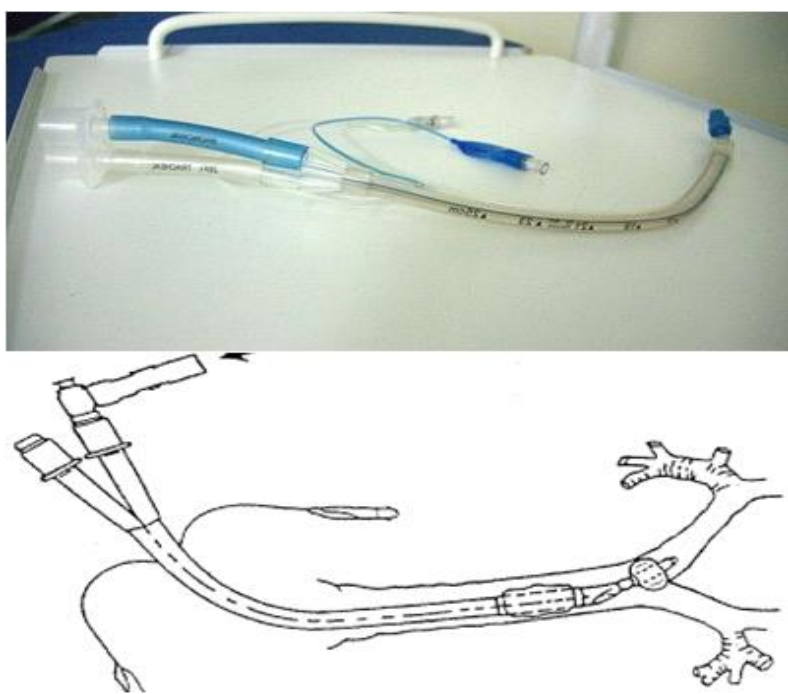

Figure 8 - Double lumen endotracheal tube (LifeSim Center for Medical Simulation and Insertion of a Double Lumen Tube,W. John Russell 17th May 2000)

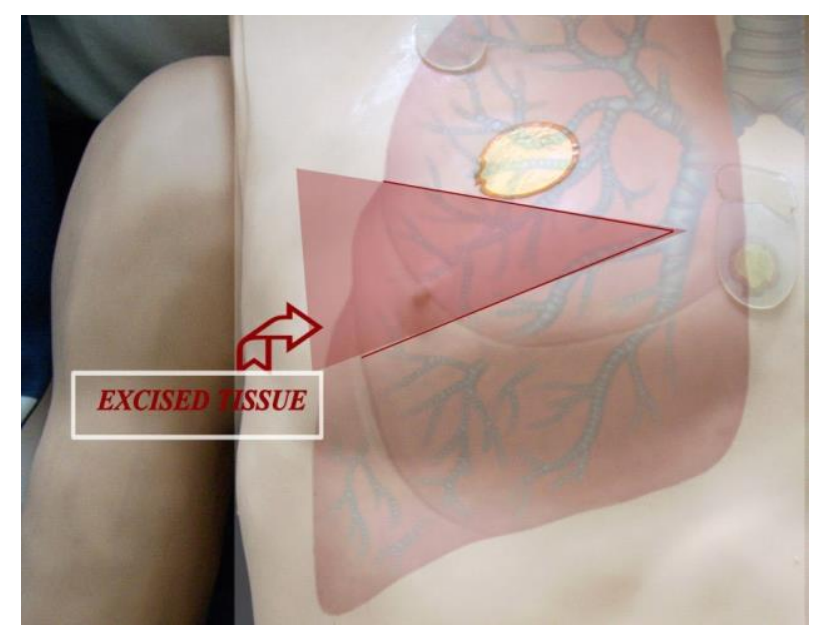

Figure 9 - Excised tissue (LifeSim Center for Medical Simulation)

The intraoperative aspect revealed the diagnosis of hydatid cyst with associated infectious process.

The cyst was extracted together with the perilesional lung parenchyma. The plans were sutured and lung ventilation was initiated, as to verify the effectiveness of haemostasis and of aerostasis (Figure 9).

A drainage tube was introduced into the pleural cavity. The drainage tube was connected to a passive vacuum system Beclaire for 4 days. 
The post-surgery evolution was good, without surgical complications.

\section{Discussions}

Hydatid lung symptoms can vary greatly, from superinfection cyst symptoms of acute fever and chest pain, to vomica with bronchial tree rupture, or pneumothorax with dyspnoea and acute pain. The most common symptom remains dry cough (in proportion of $54 \%$ ) $[7,8]$. Also, the radiological and imaging appearance can vary greatly, especially because of superinfection, creating the typical appearance of a lobar pneumonia, which can lead to confusion in establishing a final diagnosis and in managing the entire case.

Although there are two methods of treating the hydatid cyst, via surgery or medication, it is commonly believed that surgical treatment is preferred for cysts larger than $2 \mathrm{~cm}$, as the presurgery treatment with Albendazole can lead, besides the death of the parasite, to an increased risk of infection by creating microfistulas at the pericystic cavity level [9]. This was confirmed by this case, which required surgical intervention after the drug proved relatively inefficient.

Besides a clear indication of surgery in the case of a hydatid cyst greater than $2 \mathrm{~cm}$, it is important that the young practitioner considers these ideas: sometimes, the treatment with Albendazole cannot be finalized because of the peculiarities of the patient, which may have side effects following the treatment, may suffer a reversible increase in transaminases due to hepatolysis and the natural evolution of the hydatid cyst, even in the presence of antiparasite chemotherapy can worsen the condition.

\section{Conclusions}

The high incidence of hydatidosis, the high costs and the complications arising from the treatment constitute elements that determines us to support the implementation of screening programs and prevention of this condition. It is also recommended that an archive of records should be created, thus keeping track of infected patients, patients under medical treatment, the times when they have to report for check-ups, and long-term monitoring the patients who underwent surgery for eradication of the infection.

\section{References}

[1]Moldovana, R., Neghinab, A. M., Calmac, C.L., Marincud, I., and Neghinac I., Human cystic echinococcosis in two south-western and centralwestern Romanian counties: A 7-year epidemiological and clinical overview.

[2]Lazar, L., (2006). Compendiu de parazitologie medicala "Parazitii in patologia umana". Editura Universitara Carol Davila.

[3]Prof. Schmidt, G., and Prof. Roberts, L. S., (1997). Foundantions Of Parasitology.

[4]Wani, R. A., Wani, I., Malik, A. A., Parray, F. Q., Wani, A. A., and Dar, A.M., Hydatid disease at unusual sites.

[5]Orlewics, Marc S., MD, and Meyers, Arlen D., MD. Double-Lumen Endotracheal Tube Placement. [6]Mitrofan,C., Aldea, A., Grigorescu, C., Jitaru, I., Moldoveanu, C., Iosep, G., and Bolog, S., Experienta noastra in tratamentul chirurgical al chistului hidatic pulmonar, Clinica Chirurgie Toracică, Iasi.

[7]Popa, V., Rezectii pulmonare atipice. Available at: http://chirurgietoracoscopica-ctva.ro/

[8]Ranjan, R., Chowdhary, P., Pandey, A., Mishra, S., and Madan, M., Recurrent Hydatid Cyst of Liver with Asymptomatic Concomitant Hydatid Cyst of Lung: An Unusual Presentation-Case Report.

[9]Nistor,C., Ciuche, A., Pantile, D., Davidescu, M., and Ranetti, A. E., The importance of drug theraphy in lung multifocal hydatidosis.

[10]Prof. Beuran, M., (2013), Curs de chirurgie pentru studenti, Vol. 1.

[11]Romanian Society of Surgery Magazine. (n.d.). Available at: http://www.revistachirurgia.ro/ 